\title{
A summary of tuberculosis in Canada, 2013
}

\author{
Gallant $V^{1}$, McGuire $M^{1}$, Ogunnaike-Cooke $S^{1}$ * \\ ${ }^{1}$ HIVIAIDS and Tuberculosis Section, Public Health Agency of Canada, Ottawa, ON \\ *Correspondence: Susanna.Ogunnaike-Cooke@phac-aspc.gc.ca
}

\begin{abstract}
Background: The Public Health Agency of Canada (PHAC) monitors active TB disease through the national collaborative Canadian Tuberculosis Reporting System (CTBRS). PHAC uses TB surveillance data and reports to monitor progress towards achieving Canada's goal of preventing and controlling the transmission of TB, as outlined in Tuberculosis Prevention and Control in Canada - A Federal Framework for Action.

Objective: To provide an overview of the preliminary number of reported active (new and re-treatment) TB cases and corresponding incidence rates in Canada for 2013.

Methods: Provincial and territorial public health authorities voluntarily submit data to the CTBRS on an annual basis for all TB cases that meet the case definition for national-level surveillance. These data are analyzed by PHAC; the results of the analysis are validated by the supplying jurisdiction and are published annually as the Tuberculosis in Canada Pre-release series.
\end{abstract}

Results: In Canada, 1,640 new active and re-treatment TB cases were reported in 2013 for an overall incidence rate of 4.7 per 100,000 population. There was little change from preceding years in the overall distribution of cases by sex and age group. Although individuals aged 25 to 34 years old represented the largest percentage of reported cases $(16 \%)$, the highest incidence rate was observed for those aged 75 years or older. Incidence rates in British Columbia, Manitoba, Saskatchewan, Nunavut, and Northwest Territories were higher than the Canadian rate, with Nunavut showing the highest incidence rate. Foreign-born individuals continue to account for the majority of reported TB cases, but the incidence rate per 100,000 population remains highest among Canadianborn Aboriginal people.

Conclusion: Preliminary data from 2013 indicate that there were no notable changes in the number of reported cases of TB or in the overall incidence rate in Canada when compared with previous years' data. Similarly, no changes were noted in the distribution of cases by province or territory, age group or sex.

\section{Introduction}

The Public Health Agency of Canada (PHAC) provides national leadership related to public health aspects of tuberculosis (TB) and works collaboratively with domestic and international partners to address TB prevention and control. Two of the key responsibilities of PHAC are the monitoring of and reporting on TB disease through a national surveillance system, the Canadian Tuberculosis Reporting System (CTBRS) which is a collaborative effort with the provincial and territorial ministries of health.

Every year PHAC publishes a brief summary report, Tuberculosis in Canada_Pre-release (1) which facilitates timely availability of national-level surveillance data on TB. Every three years, a comprehensive surveillance report is published on Tuberculosis in Canada (2). PHAC uses these TB surveillance data and reports to monitor progress towards achieving Canada's goal of controlling and preventing the transmission of TB, as outlined in Tuberculosis Prevention and Control in Canada-A Federal Framework for Action (3). 
This article provides an overview of the preliminary number of reported active (new and re-treatment) TB cases and corresponding incidence rates in Canada for 2013 (2).

\section{Background}

As tuberculosis is legally reportable in all provinces and territories, health care practitioners are legally required to report cases to the provincial or territorial ministry of health. TB has been notifiable in Canada since 1924, which means that it has been prioritized for monitoring at the national level; however, there are no laws in place requiring provinces or territories to report case and outcome data for surveillance purposes to the national level., However, in a cooperative effort to understand the burden of TB in Canada, provincial and territorial public health authorities voluntarily submit data on TB cases that meet the case definition for national-level surveillance (See text box below) (4).

\section{National case definition for tuberculosis (TB) and re-treatment case definition}

\section{Tuberculosis}

\section{Confirmed Case}

- Laboratory confirmed case: Cases with Mycobacterium tuberculosis complex demonstrated on culture, specificallyM. tuberculosis, M. africanum, M. canetti, M. caprae, M. microti, M. pinnipedii or M. bovis (excluding M. bovis Bacillus Calmette-Guérin [BCG] strain).

- Clinically confirmed case: In the absence of culture proof, cases clinically compatible with active TB that have, for example:

I. chest $\mathrm{X}$-ray changes compatible with active TB;

II. active non-respiratory TB (meningeal, bone, kidney, peripheral lymph nodes, etc.);

III. pathologic or post-mortem evidence of active tuberculosis;

IV. favourable response to therapeutic trial of anti-tuberculosis drugs.

\section{New and Re-treatment Cases of Tuberculosis}

- New cases

No documented evidence or adequate history of previously active tuberculosis

\section{- Re-treatment case}

I. Documented evidence or adequate history of previously active tuberculosis that was declared cured or treatment completed by current standards; and

II. At least six months have passed since the last day of previous treatment; and

III. Diagnosed with a subsequent episode of TB that meets the case definition for active TB.

OR

I. Documented evidence or adequate history of previously active TB that cannot be declared cured or treatment completed by current standards; and

II. Inactive for six months or longer after the last day of previous treatment; and

III. Diagnosed with a subsequent episode of TB that meets the case definition for active TB.

OR

I. Documented evidence or adequate history of previously active TB that cannot be declared cured or treatment completed by current standards; and

II. Inactive for six months or longer after the last day of previous treatment; and

III. Diagnosed with a subsequent episode of TB that meets the case definition for active TB.

\section{Methods}

Managed by PHAC, the CTBRS is a national case-based surveillance system which maintains selected nonnominal data on people diagnosed with active TB disease including, but not limited to, demographics (e.g., age, 
sex, origin, immigration status), and clinical, diagnostic, and treatment information. Risk factor information (e.g., HIV status) and treatment outcome details were gathered, but are not included in this article.

The data are collected either through manual completion of a standard reporting form or by electronic transmission.

TB "incidence rate" is defined here as the number of individuals newly diagnosed with active TB disease (new and re-treatment cases) within each reporting year, per 100,000 population. Rates are expressed as the number of cases reported each calendar year per 100,000 population. No statistical procedures were used for comparative analyses, nor were any statistical techniques applied to account for missing data. Incidence rates in the foreignborn population are based on population estimates derived from the Canadian census. Canadian-born nonAboriginal is calculated as the total Canadian population, minus the foreign-born population, minus the total Aboriginal population. The diagnostic classification of TB in Canada is based upon the International Classification of Diseases, 9th and 10th editions $(5,6)$.

\section{Results}

In Canada, 1,640 new active and re-treatment TB cases were reported in 2013, for an overall incidence rate of 4.7 per 100,000 population which is marginally lower than both the number of TB cases reported in $2012(n=1,699)$ and the incidence rate for 2012 (4.9 per 100,000 population). The annual TB incidence rate for Canada has remained below 5 per 100,000 since 2007 (Figure 1).

Figure 1: Reported number of tuberculosis cases and incidence rate per 100,000 population, Canada, 2003-2013

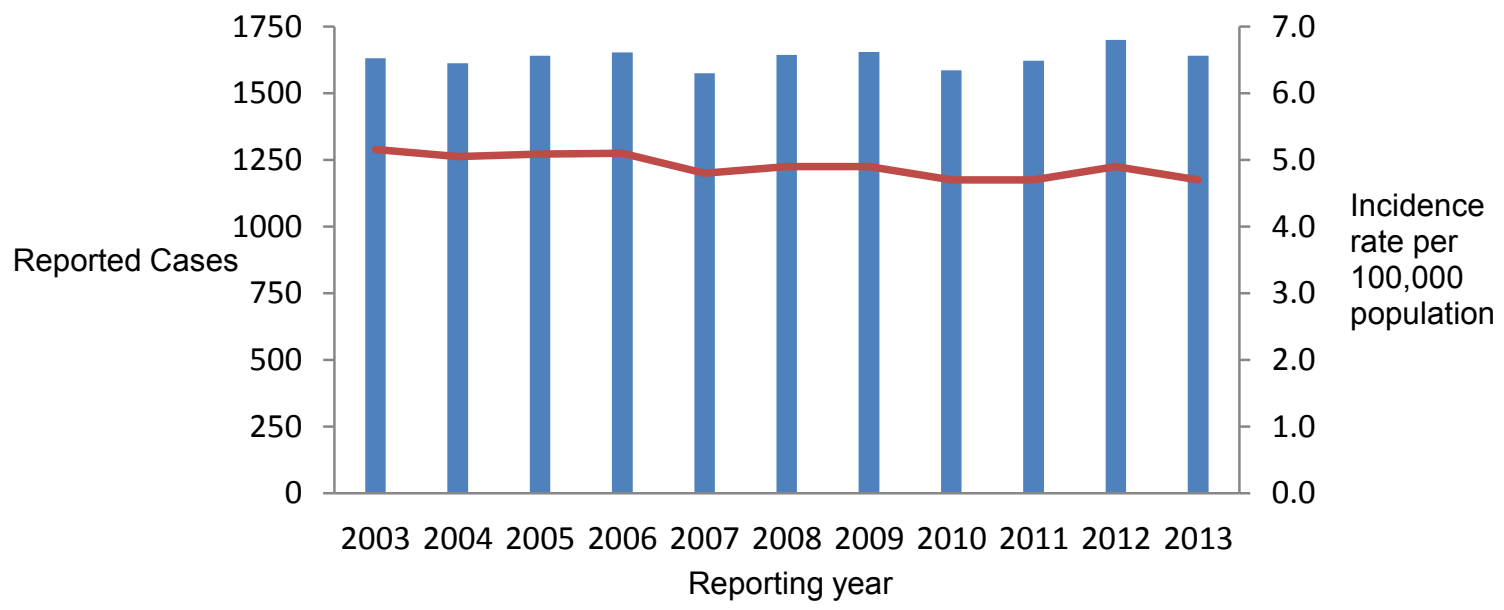

Active TB disease is classified as either respiratory or non-respiratory. Respiratory TB includes pulmonary TB, TB of the pleura, the intrathoracic or mediastinal lymph nodes, or of the larynx, nasopharynx, nose or sinuses. Nonrespiratory TB refers to all other disease sites (3). Similar to recent years, in $2013,77 \%$ of cases were diagnosed with respiratory TB and $23 \%$ with non-respiratory TB (miliary, central nervous system, peripheral lymph nodes, and other).

\section{Age and sex distribution}

In 2013 as in previous years, males accounted for the majority of reported cases at $55 \%$ for an overall incidence rate of 5.2 per 100,000 male population. In comparison, females accounted for $45 \%$ of all reported cases for a rate of 4.2 per 100,000 female population.

In 2013, individuals aged 25 to 34 years old represented the largest percentage of reported cases at $16 \%$. Seven percent of reported cases were less than 15 years of age. The highest incidence rate was observed for those aged 75 years and older, at 9.3 per 100,000 population (Figure 2). 
Figure 2: Tuberculosis incidence rate per 100,000 population, by age group, Canada, 2013

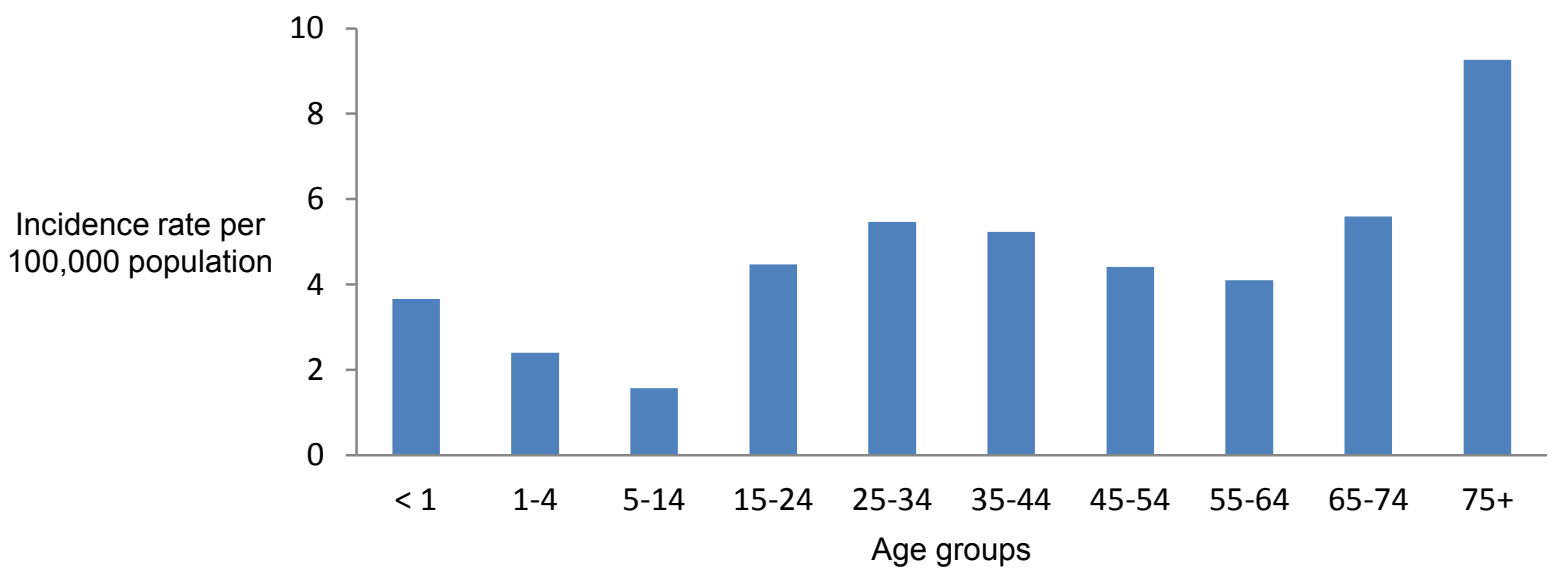

\section{Geographic distribution}

In 2013, there were no TB cases reported in Prince Edward Island. The incidence rates in the other Atlantic Provinces, as well as in Alberta, Ontario and Quebec, were all equivalent to or below the Canadian rate of 4.7 per 100,000 population (Figure 3). The incidence rates in British Columbia, Manitoba, Saskatchewan, Nunavut, Northwest Territories, and Yukon were higher than the Canadian rate. Nunavut continued to show the highest incidence rate at 143.3 per 100,000 population. Since 2004, the incidence rate in Nunavut has remained the highest of any region in Canada, peaking in 2010 at 299.8 per 100,000 population. The 2013 incidence rate for Nunavut was lower than the 2012 rate of 230.5 per 100,000 population; this drop in the rate corresponded to 29 fewer cases reported in 2013 compared with 2012.

Figure 3: Tuberculosis incidence rate per 100,000 population by province/territory, Canada, 2013

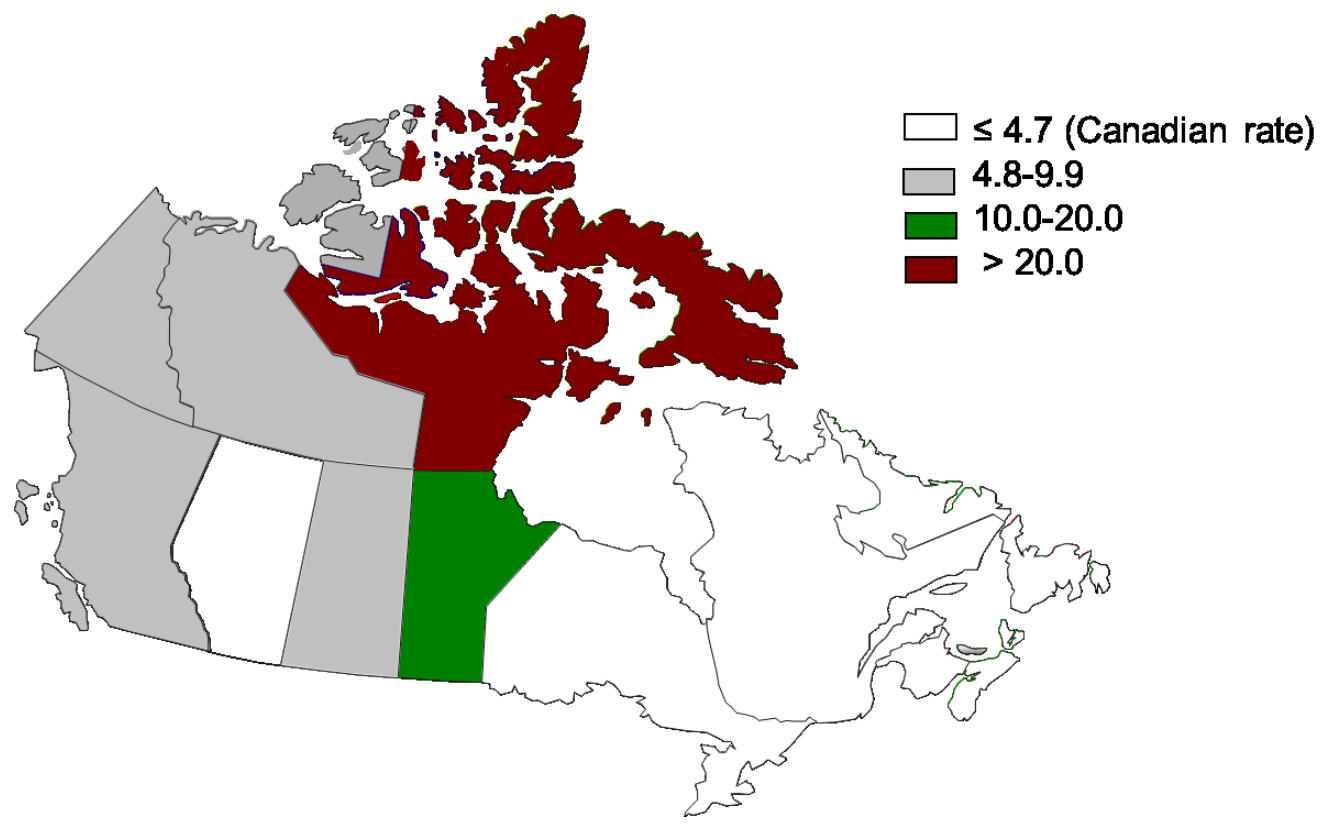




\section{Distribution by origin}

Foreign-born individuals and Canadian-born Aboriginal people continued to be disproportionately represented among reported cases of TB in 2013. Data on origin was available for 1,611 (98\%) of the 1,640 cases reported in 2013. The foreign-born population, which represented approximately $22 \%$ of the total Canadian population in 2013 , accounted for $71 \%$ of reported cases of known origin $(n=1,146)$, with a corresponding incidence rate of 14.8 per 100,000 population. Canadian-born Aboriginal people made up 4\% of the total Canadian population in 2013 but accounted for $19 \%(n=309)$ of reported cases of known origin, with a corresponding incidence rate of 19.9 per 100,000 population. Canadian-born non-Aboriginal people accounted for the lowest percentage of reported cases at $10 \%(n=155)$, with a corresponding incidence rate of 0.6 per 100,000 population (Figure 4$)$.

Figure 4: Percentage of reported tuberculosis cases by origin, Canada, 2003-2013

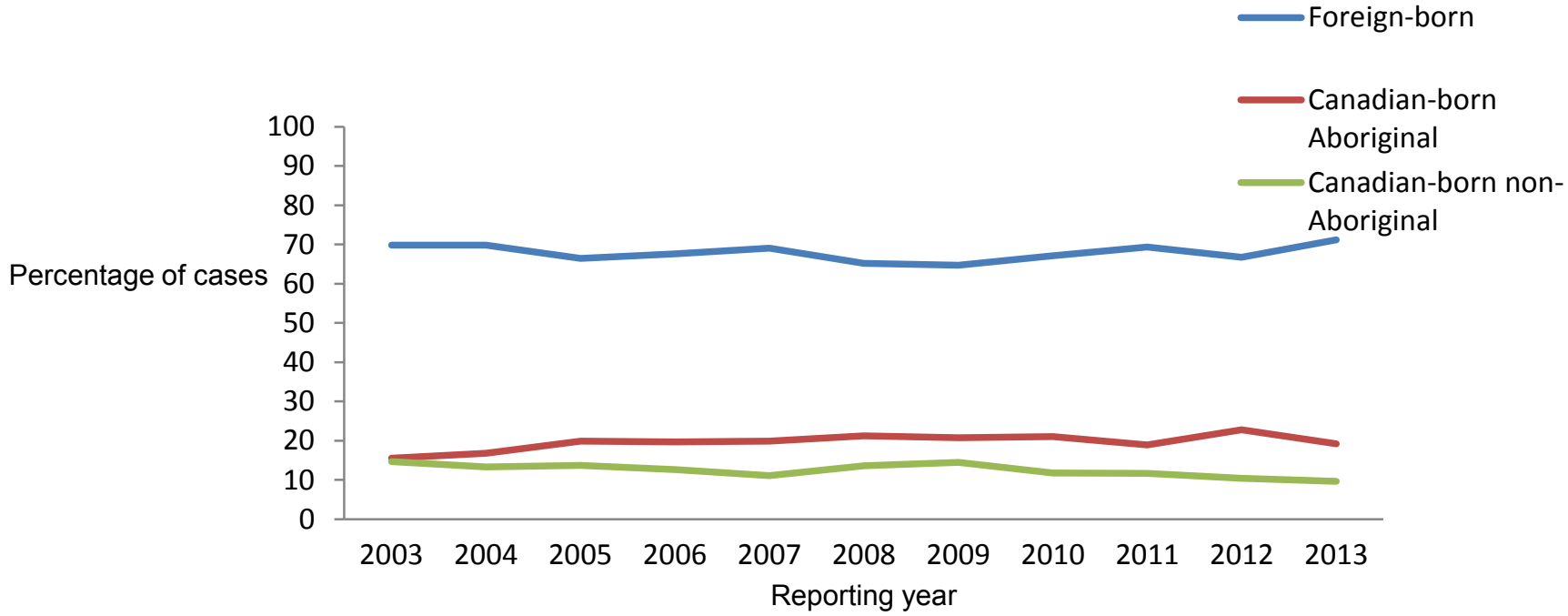

Canadian-born Aboriginal people comprise three distinct populations: First Nations, Inuit, and Métis. In 2013, of the 309 reported Canadian-born Aboriginal cases, 200 (65\%) were First Nations, 91 (29\%) were Inuit, and 18 $(6 \%)$ were Métis. The incidence rate among the Métis was 3.3 per 100,000 population, which was lower than the overall Canadian incidence rate of 4.7 per 100,000 population. By comparison, the incidence rate in First Nations, at 21.8 per 100,000 population, was almost five times the overall Canadian rate. The highest incidence rate, however, was in Inuit, at 154.2 per 100,000 population.

\section{Discussion}

Overall, Canada has one of the lowest overall TB rates in the world. Compared with 2012, preliminary data from 2013 indicate that there were no notable changes in the number of reported cases of TB or in the overall incidence rate in Canada. Similarly, no changes were noted in the distribution of cases by province or territory, or by age group or sex. Foreign-born individuals continued to account for the majority of reported TB cases, but the incidence rate per 100,000 population remained highest among Canadian-born Aboriginal people.

There are several factors that need to be considered when interpreting this data. The CTBRS is a passive surveillance system relying on data collected retrospectively from medical and laboratory records. As a result, it is difficult to ascertain whether all people with TB disease are being identified. However, the WHO estimates that Canada's surveillance system has a case detection rate of $92 \%$ with a range of uncertainty of $85 \%$ to $100 \%(7)$.

The surveillance data in this report may be subject to the occasional coding, reporting and processing errors. In general, for the reported cases data on demographic, clinical and laboratory variables are complete for over $95 \%$ of reported cases. Some provincial and territorial TB program reporting systems are unable to separately report cases that are First Nations without status from cases that are Métis. Therefore, incidence rates for the Métis should be interpreted with caution. 
Finally, the findings from this analysis are provisional. Updated case counts and incidence rates for 2013 will be presented in the next iteration of the Tuberculosis in Canada surveillance report.

In conclusion, a comprehensive understanding of the epidemiological trends of TB across Canada is integral to monitoring progress toward the goal of a reduced national TB burden. As the epidemiology of TB in Canada and the world evolves, PHAC will continue to work with its partners to improve the quality and nature of the data and report on it in a timely manner.

\section{Acknowledgments}

The Surveillance and Epidemiology Division at the Public Health Agency of Canada would like to acknowledge the provincial and territorial tuberculosis programs and their teams for their contribution to and participation in the Canadian Tuberculosis Reporting System (CTBRS).

\section{Conflict of interest}

None

\section{Funding}

This work was supported by the Public Health Agency of Canada.

\section{References}

(1) Public Health Agency of Canada. Tuberculosis in Canada 2012. Ottawa (Canada): Minister of Public Works and Government Services Canada; 2015.

http://www.phac-aspc.gc.ca/tbpc-latb/pubs/tbcan12pre/index-en.php

(2) Public Health Agency of Canada. Tuberculosis in Canada 2013—Pre-release. Ottawa, ON: Minister of Public Works and Government Services Canada; 2015.

http://www.phac-aspc.gc.ca/tbpc-latb/pubs/tbcan13pre/index-eng.php

(3) Public Health Agency of Canada. Tuberculosis Prevention and Control in Canada-A Federal Framework for Action. Ottawa, ON: Minister of Public Works and Government Services Canada; 2014.

http://www.phac-aspc.gc.ca/tbpc-latb/pubs/tpc-pct/index-eng.php

(4) Menzies R, Wong T, editors. Ottawa, ON: Minister of Health; 2013. Canadian Tuberculosis Standards. 7th ed. Canadian Thoracic Society, Canadian Lung Association Public Health Agency of Canada, http://www.lignesdirectricesrespiratoires.ca/normes-antituberculeuse-2013

(5) Canadian Institute for Health Information (CIHI). Canadian Coding Standards for ICD-10-CA and CCI. Ottawa, ON: CIHI; 2003.

https://secure.cihi.ca/estore/productSeries.htm?locale=fr\&pc=PCC189

(6) World Health Organization (WHO). Manual of the 34th international classification of diseases, injuries, and causes of death, based on the recommendations of the ninth revision conference, 1975, and adopted by the twenty-ninth World Health Assembly. Geneva: WHO; 1977.

(7) World Health Organization. Global tuberculosis report 2014. http://www.who.int/tb/publications/global_report/en/ 